\title{
Study of the Multi Pixel Photon Counter for the ILC Scintillator-Strip Calorimeter
}

\author{
Yuji Sudo* \\ Graduate School of Pure and Applied Sciences, University of Tsukuba \\ E-mail: sudo@hep.px.tsukuba.ac.jp \\ Scintillator-ECAL Group for ILC, \\ KEKDTP Photon Sensor Group
}

For the ILC experiment, we are developing and studying a finely segmented scintillator-strip calorimeter, ScECal. The Multi-Pixel Photon Counter (MPPC), manufactured by Hamamatsu Photonics, is a prime candidate for the photon sensor. Currently, we are developing and studying the 1600-pixel MPPC with $1 \times 1 \mathrm{~mm}^{2}$ sensor area. In this proceeding, we report the performance of this MPPC. Since the recovery time of the 1600-pixel MPPC is known to be short (about $4 \mathrm{~ns}$ ), each pixel can fire multiple times for the light from a scintillator-strip through wavelengthshifting fiber. Although this effect is useful, the total response of the MPPC is saturated for high intensity light. Therefore, a large number of pixels is still necessary for the ScECal readout. Understanding radiation tolerance of the MPPC is important for the ILC experiment. We have irradiated the MPPC with gamma-rays and neutrons to investigate the radiation damage. As a result of the irradiation, although the dark noise increased by irradiation, the MPPC is still working as a photon-counting device.

International Workshop on New Photon Detectors PD09

June 24-26 2009

Shinshu University Matsumoto Japan

\footnotetext{
*Speaker.
} 


\section{Introduction}

International Linear Collider experiment (ILC) is the next generation linear collider which has center of mass energy of $500 \sim 1000 \mathrm{GeV}$. ILC is expected to play an important role in the high energy physics. For the ILC experiment, three detector concepts are currently proposed. We are developing and studying the calorimeter for the ILD [1] which is one of the three detector concepts. In $e^{+} e^{-}$annihillation at ILC, many particles (Higgs, SUSY, $W, Z, t \bar{t}$, etc) are generated and decay to some multi-jet final states. Therefore, precise jet energy measurement is crucial.

Particle flow algorithm (PFA) is a powerful method for precise jet-energy mesurement [2]. Key of the PFA is separation of charged and neutral particles in a jet, in the calorimeter. In the PFA, charged particles $(\sim 60 \%$ in a jet) are measured by the central tracker. Photons $(\sim 30 \%)$ are measured by the electromagnetic calorimeter. Photons are mainly made from $\pi^{0}$ decays. Neutral hadrons (mainly $K_{L}^{0}, \sim 10 \%$ ) are measured by the calorimeter. Total energy of a jet is calculated by summation of momenta of charged particles and energy of neutral particles. For the PFA, separation of jet particles in the calorimeter is indispensable.

We are developing and studying a scintillator-strip electromagnetic calorimeter ( $\mathrm{ScECal}$ ) for the ILD. The ScECal is a sampling calorimeter with a tungsten and scintillator sandwich structure. For the ScECal readout, we use the scintillator strips with wavelength shifting fibers. The PFA requires fine granularity for the calorimeter. Therefore we employ well-established plastic scintillator which costs us very low. Layer by layer, scintillator strips are orthogonally arrayed to achieve fine granularity. For the fine segmentation, we need a large number of readout channels which are about 5 millions for the electromagnetic calorimeter. In addition, the calorimeter will be installed in 3.5 T solenoid. Therefore the Multi-Pixel Photon Counter (MPPC), manufactured by Hamamatsu Photonics, is feasible for the ILD calorimeter readout. Currently, we are developing and studying the 1600-pixel MPPC with $1 \times 1 \mathrm{~mm}^{2}$ sensor area.

\section{Response curve of the 1600-pixel MPPC}

The response of the MPPC can exceed 1600 pixels by recovery of pixels which have been fired at least once. The response curve depends on the input light pulse shape because the recovery time constant is very short (about $4 \mathrm{~ns}$ ) [3]. We have to understand the saturation effect to measure the correct energy of particles into the calorimeter. In this time, we measure the response curve for the beam test of prototype of the ScECal. We use a MPPC and a scintillator-strip with wavelengthshifting fiber. This setup is the same combination for the beam test (Figure 1 left). For the light source, we use a pico-second light pulser which has about $400 \mathrm{~nm}$ wavelength and $60 \mathrm{ps}$ pulse width. The response curve is able to be fitted using function (2.1) (Figure 11 right) :

$$
N_{\text {fired }}=N_{\text {pixel }}\left\{1-\exp \left(\frac{-N_{\text {true }}}{N_{\text {pixel }}}\right)\right\}
$$

where $N_{\text {fired }}$ is the observed number of fired pixels of MPPC, $N_{\text {true }}$ is the true light yield and $N_{\text {pixel }}$ is the effective number of pixels. From this measurement, we obtain a result that the $N_{\text {pixel }}$ is 1.5 times larger than the real number of pixels (1600 pixels), because of the recovery effect for each pixel. 

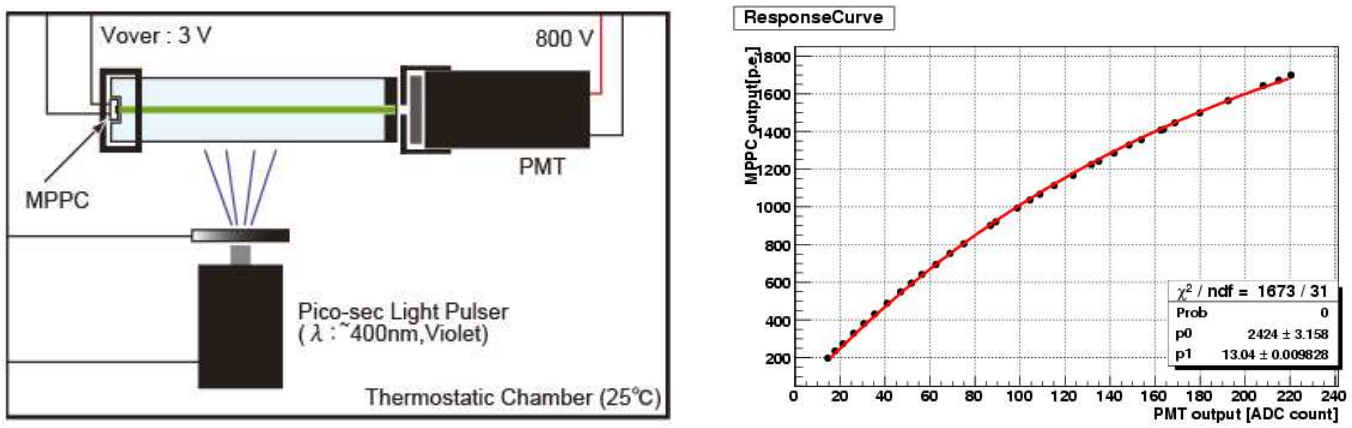

Figure 1: The left figure shows the setup of the measurement for response curve. The right plot shows the measured data (black points) which is fitted with function (2.1) (red line).

We make a simulation for the response of MPPC to confirm our understanding. To make the simulation, we have to evaluate quantitatively the following parameters for MPPC : recovery time constant, photon detection effeciency, probability of cross-talk and after pulsing. We have already had these values from our measurements [3, 那. It is also important to take the pulse shape of the input light into acoount. We compare simulated MPPC outputs to real data using LED for the light source. The left plot of Figure 2 shows the response for low intensity input light yield with noise which comes from electronics. In this plot, the horizontal axis is MPPC output in unit of ADC count and the vertical axis shows number of events. The right plot of Figure 2 shows the response curve for high intensity input light yield. In this plot, the horizontal axis is input light yield and the vertical axis shows MPPC output in unit of fired pixel. The simulation is in good agreement with the measured data. For further improvement, we need to optimize the shape of the input light pulse and to precisely evaluate properties of the MPPC.
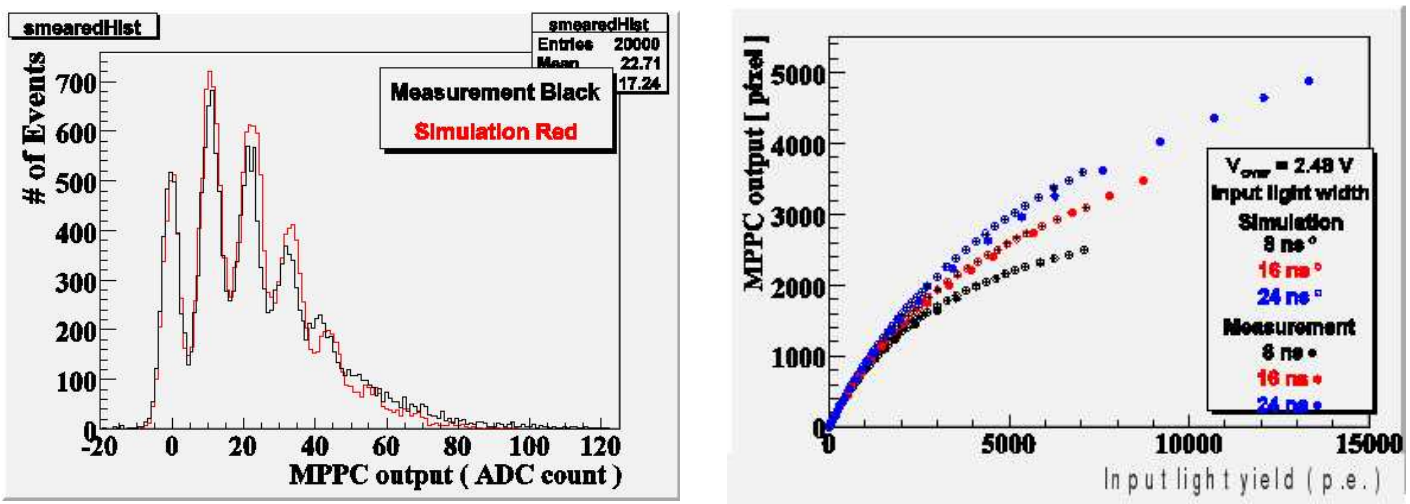

Figure 2: These plots show measured data and simulated data for outputs of MPPC. The left plot shows ADC distribution for a few photons. The right plot shows response curve of the MPPC. In the right plot, the horizontal axis is input light yield in unit of photoelectron (p.e.).

\section{Study of the irradiation effect for 1600-pixel MPPC}

It is important to know radiation tolerance in order to estimate the life time of the calorimeter under the environment at ILC. We make two different irradiation tests for the MPPC. The first test 
is $\gamma$-ray irradiation with ${ }^{60} \mathrm{Co}$ source. The second test is neutron irradiation with a reactor YAYOI at the university of Tokyo. For the $\gamma$-ray, the prospective damage is charge accumulation on the oxidized layer. On the other hand, for the neutron irradiation, increasing lattice defect in silicon bulk is expected.

\section{1 $\gamma$-ray irradiation}

We have a $\gamma$-ray irradiation test with ${ }^{60} \mathrm{Co}$ source at Tokyo Institute of Technology. We test with three dose amounts of 30, 60 and $120 \mathrm{~Gy}$. Each sample is irradiated with $10 \mathrm{~Gy} / \mathrm{hour}$.

During irradiation, we measure the current with applied voltage. For 30 and $60 \mathrm{~Gy}$ irradiated samples, the current are runnning constantly because MPPC samples are just counting the number of $\gamma$-rays from ${ }^{60} \mathrm{Co}$ source. As it is found in Figure 3, $120 \mathrm{~Gy}$ irradiated sample shows a drastic change at about $90 \mathrm{~Gy}$. It seems that some fatal damage has occurred at $90 \mathrm{~Gy}$.

After $\gamma$-ray irradiation, we measure leakage current, noise rate, probability of corss-talk, gain and response curve. The leakage current and noise rate (Figure $\$$ ) are increased depending on total amount of irradiation. For ScECal, the noise rate should be less than $1 \mathrm{MHz}$, while $60 \mathrm{~Gy}$ irradiated sample exceeds $1 \mathrm{MHz}$ with high applied voltage. There seems no significant change on cross-talk probability by irradiation. For the 120 Gy sample, we can't measure the noise rate and cross-talk probability because dark count rate is too high. Gain and response curve (Figure F) haven't changed by $\gamma$-ray irradiation. We take infrared pictures to see the hot spots which always let out noise (Figure 6). It seems that the number of hot spots has increased by $\gamma$-ray irradiation. The hot spots only appear on the thick oxidized part which are along the bias line.

Although noise rate has increaced by $\gamma$-ray irradiation, the MPPC is still working as a photoncounting device.

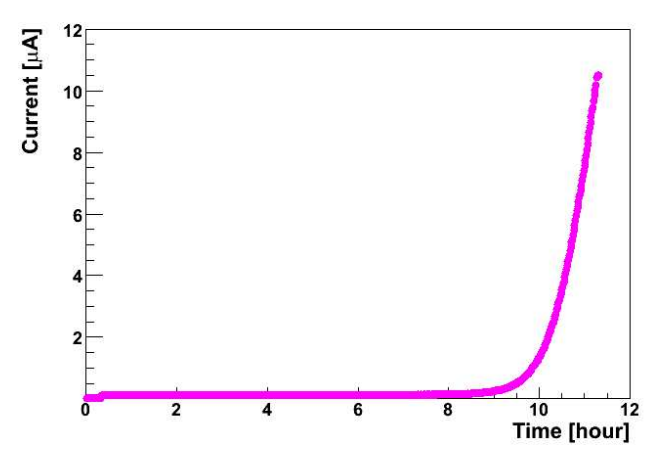

Figure 3: This plot shows the current during $\gamma$ ray irradiation with ${ }^{60} \mathrm{Co}$.

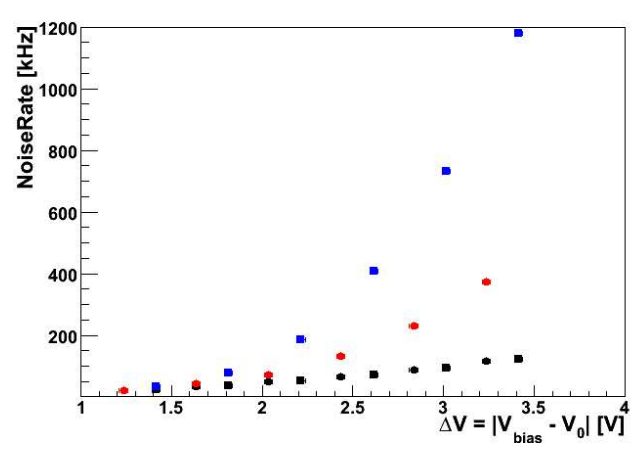

Figure 4: This plot shows the result of measurement for noise rate. Black, red and blue points show rusults of before irradiation, after $30 \mathrm{~Gy}$ and 60 Gy irradiation, respectively.

\subsection{Neutron irradiation}

We have a neutron irradiation test with four amounts of dose, namely, $1.2 \times 10^{8}, 1.2 \times 10^{9}$, $1.2 \times 10^{10}, 1.2 \times 10^{11}$ neutron $/ \mathrm{cm}^{2}$, respectively. These numbers are converted to the dose of $1 \mathrm{MeV}$ 


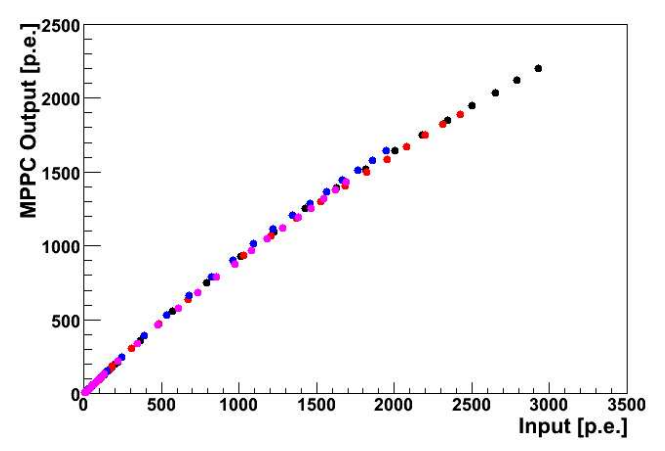

Figure 5: This plot shows the result of measurement for response curve. Black, red, blue and pink points show rusults of before irradiation, after 30 Gy, 60 Gy and 120 Gy irradiation, respectively.

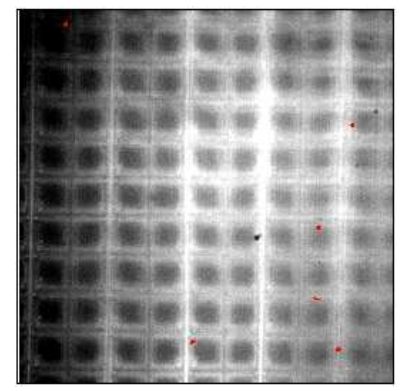

Figure 6: This is the infrared picture. Red points show hot spots.

neutrons. We measure some properties before irradiation and after 2 days and after 40 days from irradiation.

As the results of neutron irradiation, the leakage current and noise rate have increased (Figure 7 left). We observe that the leakage current and noise rate have decreased after 40 days from those measured immidiately after irradiation. An annealing effect is observed for samples which are irradiated more than $10^{9}$ neutron $/ \mathrm{cm}^{2}$. But for $1.2 \times 10^{9}$ neutron $/ \mathrm{cm}^{2}$ sample still has higher noise rate than $1 \mathrm{MHz}$. On the other hand, cross-talk probability, gain, shape of response curve and resolution of output are not changed. These results show that MPPC is still working after neutron irradiation. We also take infrared pictures to see the hot spots (Figure 7 right). In this case, hot spots appear only inside of the sensitive area.
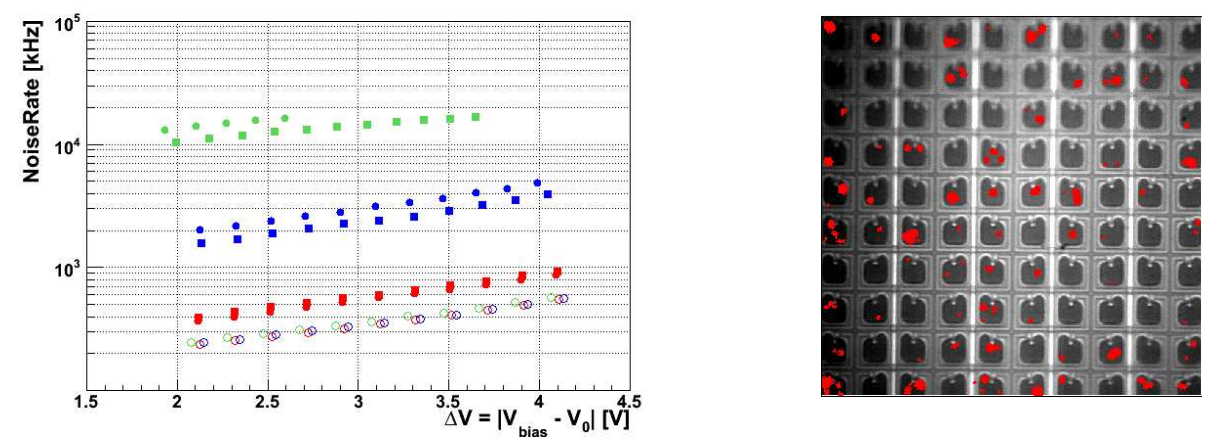

Figure 7: The left plot shows the result of noise rate measurement. Black, red, blue and green points show results of before irradiation, after $10^{8}, 10^{9}$, and $10^{10}$ neutron $/ \mathrm{cm}^{2}$ irradiation, respectively. Opening circles, circles and squares show measured points before irradiation, after 2 days and after 40 days. The right figure shows the infrared picture for the $10^{11}$ neutron $/ \mathrm{cm}^{2}$ irradiated sample.

\section{Summary}

We have to understand the saturation effect of 1600-pixel MPPC to measure correct energy of 
particles into the calorimeter. We can fit the response curve by function 2.1. The simulation for the response of the MPPC is in good agreement with the measured data. This simulation is based on the measurement for several properties.

We have two different irradiation tests with $\gamma$-ray and neutron. As results of both tests, there seem no significant changes on the cross-talk probability, gain and response curve. But hot spots appear only along bias lines after $\gamma$-ray irradiation. On the other hand, they are seen only inside of the sensitive area after neutron irradiation. Although the dark noise is increased by irradiation, the MPPC is still working as photon-counting device.

\section{References}

[1] "ILC Reference Design Report", http://ilcdoc.linearcollider.org/record/6321/files/ILC_RDR_Volume_4-Detectors.pdf.

[2] T. Yosioka、 Particle Flow Algorithm for GLD, Proceedings of 2005 ALCPG \& ILC Workshops, Snowmass. http://www-conf.slac.stanford.edu/econf/C0508141/proc/papers/ALCPG1102.PDF.

[3] Satoru Uozumi, in proceedings of International Workshop on New Photon Detectors PD07. POS (PD07) 022 .

[4] Hideyuki Oide, Takuro Murase, Hidetoshi Otono, Satoru Yamashita, arXiv:0811.1402. 